\title{
Bilateral mammary duct ectasia induced by sulpiride-associated hyperprolactinemia: A case report
}

\author{
YIZI CONG*, HAIDONG ZOU*, GUANGDONG QIAO, JUN LIN, XINGMIAO WANG, \\ XIAOHUI LI, YALUN LI and SHIGUANG ZHU \\ Department of Breast Surgery, Yantai Yuhuangding Hospital Affiliated to Medical College of Qingdao University, \\ Yantai, Shandong 264400, P.R. China
}

Received July 10, 2014; Accepted February 17, 2015

DOI: $10.3892 / 01.2015 .3034$

\begin{abstract}
A 32 year old female diagnosed with schizophrenia was treated with sulpiride, trihexyphenidyl and alprazolam for 6 years. A physical examination revealed bilateral nipple retraction and a non-tender mass in the left breast, with little nipple discharge. Tests revealed high levels of carbohydrate antigen 125, serum prolactin and testosterone levels, and ultrasound revealed a number of masses in the bilateral breasts; the largest mass $(2.2 \times 1.3 \mathrm{~cm})$ was located in the left breast. A rich blood flow signal was identified around the nodule. The ducts in the bilateral breasts exhibited cystic ectasia. Multiple enlarged lymph nodes were found in the bilateral axillae. Mammography revealed thickened breast tissue without an evident mass, and calcification. A segmental mastectomy was performed and subsequent histological examination revealed multiple dilated ducts, the largest of which contained eosinophilic material. The pathological diagnosis was of breast duct dilatation. Bacterial culture and drug sensitivity analysis of the secretions from the cystic cavity revealed no bacterial growth, and an acid fast bacillus stain was negative. Extravasation of the surgical wound occurred 1 month later, and Staphylococcus epidermidis was observed using a bacterial culture. This was treated with moxifloxacin for 1 week. It was suggested that the patient should switch to a prolactin sparing antipsychotic in view of the hyperprolactinemia, however, the patient refused. After a clinical follow-up of 16 months, the wound had healed well and no palpable mass was found in the breast.
\end{abstract}

Correspondence to: Mr. Shiguang Zhu, Department of Breast Surgery, Yantai Yuhuangding Hospital Affiliated to Medical College of Qingdao University, 20 Yudong Road, Yantai, Shandong 264400, P.R. China

E-mail: ytzhushiguang@medmail.com.cn

${ }^{*}$ Contributed equally

Key words: mammary duct ectasia, sulpiride, hyperprolactinemia, schizophrenia

\section{Introduction}

Mammary ductal ectasia (MDE) (1) is an inflammatory breast disease with the pathological characteristics of dilation of the major ducts associated with intraluminal plugs of histiocytes and periductal inflammation (2). The frequency of MDE may range from $1.1 \%$ to $75 \%$, according to the diagnostic method used, which might be clinical, histopathological or necropsybased (3). MDE typically occurs in females undergoing menopause, but can also occur in younger females, males and infants. The disease usually affects mammary ducts that are large or medium in size, and is one of the most prevalent causes of bloody nipple discharge and sub-areolar masses, radiologically and clinically mimicking invasive carcinoma $(1,4,5)$. The treatment of MDE is usually conservative. Biopsy is often required if the signs and symptoms of ectasia do not disappear or indicate a tumor. When severe, duct ectasia may require repeated surgical treatments and occasionally, mastectomy (3). The etiology and pathogenesis of MDE have not been defined. One previous study described an association between the abnormal secretion of prolactin and MDE (6). The present study reports a case of bilateral MDE induced by sulpiride-associated hyperprolactinemia in a young female with long-term schizophrenia.

\section{Case report}

A 32-year-old female presented to Yantai Yuhuangding Hospital Affiliated to Medical College of Qingdao University (Yantai, Shandong, China) in 2012 with a 4-year history of a non-palpable mass in the bilateral breast initially detected by ultrasound, with mastalgia. The patient described a history of a small amount of intermittent creamy nipple discharge. The patient's menarche occurred at a normal age, but with irregular menstrual periods. The patient married at 26 -year-old and had one child, which had not been breast-fed. The patient had never smoked and did not have a family history of cancer. A diagnosis of schizophrenia was made at the age of 26 years, followed by treatment with $0.5 \mathrm{~g}$ sulpiride once a day, $2 \mathrm{mg}$ trihexyphenidyl twice a day and $0.8 \mathrm{mg}$ alprazolam every night, for six years. A dysembryoma of the ovary had also previously been removed in Yantai Yuhuangding Hospital Affiliated to Medical College of Qingdao University. 

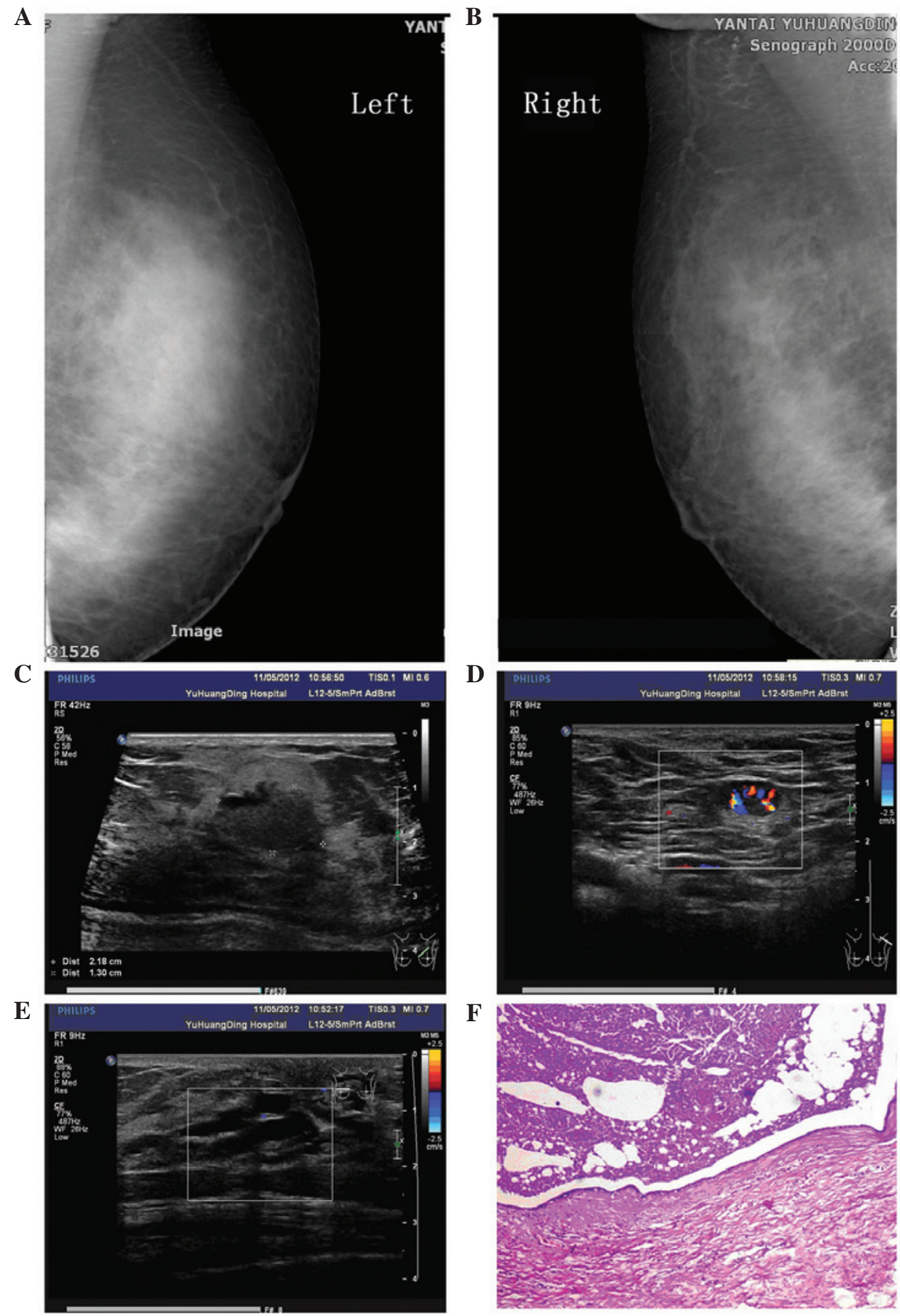

Figure 1. (A) Left and (B) right mammography revealing thickened breast tissue, without an observable mass, and sand-like calcification in the bilateral breasts. (C) Ultrasound showing the largest mass measuring $2.2 \times 1.3 \mathrm{~cm}$ located in the upper inner quadrant of the left breast, with a clear boundary and uneven echo. (D) An enlarged lymph node with a rich blood flow signal around the nodule in the left axilla. (E) A duct of the bilateral breasts exhibiting cystic ectasia. (F) Pathological sample showing breast duct dilatation.

A physical examination revealed bilateral nipple retraction and a $1.5 \times 1.0-\mathrm{cm}$ non-tender mass in the upper internal quadrant of the left breast, with a small amount of nipple discharge. There was no evidence of axillary lymphadenopathy.

An auxiliary examination revealed a carbohydrate antigen-125 level of $73.52 \mathrm{U} / \mathrm{ml}$ (normal value, $0-35.0 \mathrm{U} / \mathrm{ml})$, a serum prolactin level of $100.6 \mathrm{ng} / \mathrm{ml}$ (normal value, $6.0-29.9 \mathrm{ng} / \mathrm{ml}$ ) and a testosterone level of $0.992 \mathrm{ng} / \mathrm{ml}$ (normal value, $0.06-0.82 \mathrm{ng} / \mathrm{ml}$ ), as detected by a Roche Cobas E601 electrochemiluminescence immunoassay analyzer (Roche Diagnostics GmbH, Mannheim, Germany).
Ultrasound examination performed 4 years previously revealed several well-defined cysts. A repeat ultrasound performed following referral to Yantai Yuhuangding Hospital Affiliated to Medical College of Qingdao University revealed several masses in the bilateral breasts. The largest mass, measuring $2.2 \times 1.3 \mathrm{~cm}$, was located in the upper inner quadrant of the left breast, with a clear boundary and uneven echo. Color Doppler flow imaging revealed a rich blood flow signal around the nodule. The ducts in the bilateral breasts exhibited cystic ectasia and a maximum duct size of $0.8 \times 1.8 \mathrm{~cm}$. Multiple enlarged lymph nodes were found in the bilateral 
axillae. The largest node in the right axilla was $1.6 \times 0.8 \mathrm{~cm}$ and the largest node in the left axilla was $1.4 \times 0.8 \mathrm{~cm}$. The nodes exhibited clear boundaries with an uneven echo and had not fused together (Fig. 1).

Mammography showed thickened breast tissue without an observable mass, and sand-like calcification in the bilateral breasts (Breast Imaging-Reporting and Data System classification, 0) (7). The patient refused to undergo pituitary magnetic resonance imaging. A clinical diagnosis of mammary duct ectasia and breast tumors in the bilateral breasts was formed (Fig. 1).

A segmental mastectomy was preformed in November 2012 due to the palpable mass and nipple discharge in the left breast. Macroscopic examination revealed that the duct exhibited ectasia, with multiple galactoceles and sebaceous gland cysts containing white/yellow-colored fluid. The gland was fibrous and hard. Similar fluid was drained from the peri-areolar ducts once the lesion had been excised. Histological examination revealed multiple dilated ducts, the largest measuring $4.5 \times 3.5 \mathrm{~cm}$ and containing eosinophilic material. The duct epithelium was flattened or lost in places. The breast lobule was atrophic. The interstitial fibrous tissue and lymphoid exhibited hyperplasia with formation of lymphoid follicles. A large amount of infiltrative foam cells was also found. The subsequent pathological diagnosis was of breast duct dilatation (Fig. 1).

Next, a bacterial culture and drug sensitivity analysis was performed. The secretions were inoculated into a blood plate and a Jimmy Kang Kai plate, and then cultured in an incubator for $24 \mathrm{~h}$ at $37^{\circ} \mathrm{C}$. If bacterial growth was detected, the colonies were selected out and tested by a VITEK 2 Compact system (bioMérieux, Ltd., Marcy l'Etoile, France) for drug sensitivity. Bacterial culture and drug sensitivity analysis of the secretions from the cystic cavity revealed no bacterial growth after being cultured for two days, and an acid-fast bacillus smear test was negative. The surgical incision was beginning to heal, however, extravasation of the wound occurred one month later. Saphylococcus epidermidis was observed using a bacterial culture. The bacteria were sensitive to moxifloxacin, which was used for one week for wound healing.

We suggested that the patient should switch to a prolactin-sparing antipsychotic in view of the hyperprolactinemia. However, the patient rejected the suggestion. After a clinical follow-up of 16 months, the wound had healed well and no palpable mass was found in the breast.

The study protocol was approved by the Human Ethical Committee of the Yantai Yuhuangding Hospital Affiliated to Medical College of Qingdao University. Informed consent was obtained from the patient prior to the surgery and the examination of the specimens.

\section{Discussion}

MDE is an inflammatory breast disease associated with inflammation and periductal fibrosis of varying degrees (8). The disease has also been known as comedomastitis, plasma cell mastitis and cholesterol granuloma. Duct dilatation is usually asymptomatic in the initial phases (9). When symptomatic, the most common clinical presentation is mammary secretion (10). In the majority of cases, no palpable abnormalities are present, however, in later stages, painful sub-areolar masses with associated skin retraction occur, which can be misdiagnosed as infiltrative carcinoma. The diagnosis of MDE is usually clinical. Mammography usually identifies irregular thickening of the sub-areolar breast tissue and microcalcifications, which can mimic a carcinoma (11). Ultrasonography allows the diagnosis and measurement of duct diameters of $>5 \mathrm{~mm}$ (12). Ductography usually identifies ductal dilatation with multifocal luminal obstruction in a beaded pattern, but is limited as a diagnostic method for MDE (13). Histopathologically, the diagnosis requires the identification of peri-ductal inflammation. Circumferential deposits of lymphocytes, plasma cells or foamy histiocytes are usually found. The initial phase of MDE is characterized by the presence of dilatation of the duct terminals (14). Following this, the inflammatory process is replaced by fibrosis. The approach towards MDE is usually conservative. Duct ectasia occasionally improves without treatment or with the use of warm compresses and antibiotics. Biopsy is usually required if the signs and symptoms of ectasia do not disappear, and the abnormal duct can be removed surgically. Surgery is also reserved for cases associated with suspected malignant abnormalities. Duct excision usually provides good results for symptomatic duct ectasia. In its most severe form, duct ectasia may require repeated surgical treatments and occasionally, mastectomy (9).

Two theories exist with regard to the pathogenesis of MDE (15). First, the primary pathological process is believed to be involutional, with atrophy of the ducts and glands, followed by dilation of the ducts, leading to the inactivity of secretion, duct rupture and inflammation. Second, the causative event is proposed to be an inflammatory process; periductal inflammation being the underlying abnormality, followed by duct sclerosis, obliteration and ectasia. However, the etiology of duct ectasia is unknown. Predisposing factors include squamous metaplasia of the terminal duct epithelium, phenothiazine treatment, cigarette smoking, bacterial growth and hyperprolactinemia. Rahal et al (16) suggested that tobacco smoking is a risk factor for ductal ectasia, while a history of breast-feeding, hormonal contraceptive use, abortions and breast abscesses has no association. A study by Dixon et al (17) indicated that smoking is a significant risk factor for periductal mastitis, but not for duct ectasia. One previous study hypothesized that duct ectasia may be due to bacterial contamination by aerobic and anaerobic agents (16). Certain reports have recorded the presence of bacterial growth in infants (18) and adults (10) with duct ectasia. In the present case, Staphylococcus epidermidis was observed using a bacterial culture. However, this was not enough to be believed to be etiological, and bacterial infection is more likely to be secondary rather than a primary cause. Another previous study (6) described an association between abnormal prolactin secretion and MDE. Shousha et al (19) also concluded that an association existed between certain hypothalamic/pituitary disorders, possibly associated with prolactin secretion and mammary duct ectasia development in postmenopausal patients.

Antipsychotic drugs are the foundation of treatment for schizophrenia, a chronic and debilitating psychotic mental disorder. All conventional antipsychotic drugs block D2 receptors on lactotroph cells, thereby removing the main inhibitory effect on prolactin secretion. With an occurrence 
rate of $40-50 \%$, hyperprolactinemia is one of the most common side-effects associated with antipsychotics. Higher prolactin levels are a consequence of longer exposure to high-dose antipsychotics, and are particularly evident during treatment with older antipsychotics or with risperidone, sulpiride or amisulpride. Hyperprolactinemia may result in galactorrhea, enlargement of the breasts, ovarian dysfunction, reduced libido, reduced vaginal lubrication, dyspareunia, infertility, and atrophic changes in the urethra and vaginal mucosa. Serum prolactin levels are usually measured in patients on antipsychotic drugs when the clinical indicators of galactorrhea, amenorrhea and loss of libido or impotence are present. By analogy with prolactinoma-induced symptoms, increased levels of $>1,500 \mathrm{mU} / 1$ and particularly $>3,000 \mathrm{mU} / 1$ are likely to cause symptoms. Decreasing the dose of antipsychotic should be considered if it is unnecessarily high. Hyperprolactinemia is rapidly reversed following the discontinuation of amisulpride treatment (20) or once the patient has been switched to a prolactin-sparing antipsychotic, such as quetiapine (21), olanzapine, ziprasidone or aripiprazole. The addition of the partial agonist aripiprazole (22), including the use of dopamine agonists, to the treatment was an alternative approach. However, thi is associated with a risk of worsening or inducing psychosis, which would require specialist services. Carmoxirole is also able to reduce the hyperprolactinemia induced by amisulpride without altering its main effect (23). However, bromocriptine appears to destroy the main effect of amisulpride at a dose that reduces amisulpride-induced hyperprolactinemia, and is therefore not a suitable treatment $(21,24)$.

In the present case, we hypothesize that there was an association between sulpiride-induced prolactin secretion and the development of mammary duct ectasia. First, the patient was treated with sulpiride in high doses for 6 years, during which, bilateral creamy nipple discharge was observed. Second, the serum prolactin level in the patient was extremely $\operatorname{high}(2,132.72 \mathrm{mU} / \mathrm{l})$. There have been no reports on the use of trihexyphenidyl or alprazolam to induce hyperprolactinemia. Third, ultrasonography revealed multiple ducts that exhibited cystic ectasia in the bilateral breasts rather than a localized lesion. We believe that this was the result of the changes in the serum hormone levels. Fourth, one previous study (6) described an association between the abnormal secretion of prolactin and MDE. The possible mechanism behind this is through the inspissated secretion leading to chronic inflammation, fibrosis and the persistence of ductal dilatation. Bilateral nipple retraction is also one of the causes of MDE. The delay in the development of ectasia can be explained by the fact that the disease process probably does not start until atrophic changes have occurred in the breast.

In summary, the present study reports the case of a long-term schizophrenia patient with a high level of prolactin and bilateral MDE. We hypothesize that there is an association between the two conditions, although the specific mechanisms involved remain unclear. In view of this, certain antipysychotics that have a lower potential for increasing prolactin levels should be considered when prescribing an antipsychotic. In the future, larger studies are required to elucidate the association between these disorders, so that the risk factors can be identified for assessment in clinical practice, thereby enabling disease prevention.

\section{References}

1. McHoney M, Munro F and Mackinlay G: Mammary duct ectasia in children: report of a short series and review of the literature. Early Hum Dev 87: 527-530, 2011.

2. Haagensen CD: Mammary-duct ectasia; a disease that may simulate carcinoma. Cancer 4: 749-761, 1951.

3. Thomas WG, Williamson RC, Davies JD and Webb AJ: The clinical syndrome of mammary duct ectasia. Br J Surg 69: 423-425, 1982.

4. Dogan BE, Ceyhan K, Tukel S, Saylisoy S and Whitman GJ: Ductal dilatation as the manifesting sign of invasive ductal carcinoma. J Ultrasound Med 24: 1413-1417, 2005.

5. Duchesne N, Skolnik S and Bilmer S: Ultrasound appearance of chronic mammary duct ectasia. Can Assoc Radiol J 56: 297-300, 2005.

6. Peters F and Schuth W: Hyperprolactinemia and nonpuerperal mastitis (duct ectasia). JAMA 261: 1618-1620, 1989.

7. D'Orsi C, Mendelson EB, Ikeda D, et al: Breast Imaging Reporting and Data System: ACR BI-RADS - breast imaging atlas. American College of Radiology; Reston, VA: 2003.

8. Leung AK and Kao CP: Mammary duct ectasia: a cause of bloody nipple discharge. J Natl Med Assoc 96: 543-545, 2004.

9. Browning J, Bigrigg A and Taylor I: Symptomatic and incidental mammary duct ectasia. J R Soc Med 79: 715-716, 1986.

10. Rahal RM, Junior RF, Reis C, Pimenta FC, Netto JC and Paulinelli RR: Prevalence of bacteria in the nipple discharge of patients with duct ectasia. Int J Clin Pract 59: 1045-1050, 2005.

11. Sweeney DJ and Wylie EJ: Mammographic appearances of mammary duct ectasia that mimic carcinoma in a screening programme. Australas Radiol 39: 18-23, 1995.

12. Rizzatto G and Chersevani R: Breast ultrasound and new technologies. Eur J Radiol 27 Suppl 2: S242-249, 1998.

13. Sakorafas GH: Nipple discharge: current diagnostic and therapeutic approaches. Cancer Treat Rev 27: 275-282, 2001.

14. Ying $\mathrm{T}, \mathrm{Li} \mathrm{Q}, \mathrm{Xu} \mathrm{L}$, Liu F and Hu B: Three-dimensional ultrasound appearance of pelvic floor in nulliparous women and pelvic organ prolapse women. Int J Med Sci 9: 894-900, 2012.

15. Wang Z, Leonard MH, Jr., Khamapirad T and Castro CY: Bilateral extensive ductitis obliterans manifested by bloody nipple discharge in a patient with long-term diabetes mellitus. Breast J 13: 599-602, 2007.

16. Rahal RM, de Freitas-Júnior R and Paulinelli RR: Risk factors for duct ectasia. Breast J 11: 262-265, 2005.

17. Dixon JM, Ravisekar O, Chetty U and Anderson TJ: Periductal mastitis and duct ectasia: different conditions with different aetiologies. Br J Surg 83: 820-822, 1996.

18. Kitahara S, Wakabayashi M, Shiba T, Nonaka K, Nonaka H and Kobayashi I: Mammary duct ectasia in children presenting bloody nipple discharge: a case in a pubertal girl. J Pediatr Surg 36: E2, 2001.

19. Shousha S, Backhouse CM, Dawson PM, Alaghband-Zadeh J and Burn I: Mammary duct ectasia and pituitary adenomas. Am J Surg Pathol 12: 130-133, 1988.

20. Paparrigopoulos T, Liappas J, Tzavellas E, Mourikis I and Soldatos C: Amisulpride-induced hyperprolactinemiais reversible following discontinuation. Prog Neuropsychopharmacol Biol Psychiatry 31: 92-96, 2007.

21. Kovács L and Kovács G: Endocrine side effects among psychiatric patients treated with antipsychotics. Neuropsychopharmacol Hung 8: 61-66, 2006 (In Hungarian).

22. Mir A, Shivakumar K, Williamson RJ, McAllister V, O'Keane V and Aitchison KJ: Change in sexual dysfunction with aripiprazole: a switching or add-on study. J Psychopharmacol 22: 244-253, 2008.

23. Marchese G, Ruiu S, Casti P, et al: Carmoxirole is able to reduce amisulpride-induced hyperprolactinemia without affecting its central effect. Eur J Pharmacol 447: 109-114, 2002.

24. Bliesener N, Yokusoglu H, Quednow BB, Klingmüller D and Kühn KU: Usefulness of bromocriptine in the treatment of amisulpride-induced hyperprolactinemia: a case report. Pharmacopsychiatry 37: 189-191, 2004. 